\title{
Monitoring the healing process of laser-induced microvascular lesions using optical-resolution photoacoustic microscopy
}

Song $\mathrm{Hu}$, Konstantin I. Maslov, Lihong V. Wang

Song Hu, Konstantin I. Maslov, Lihong V. Wang, "Monitoring the healing process of laser-induced microvascular lesions using optical-resolution photoacoustic microscopy," Proc. SPIE 7177, Photons Plus Ultrasound: Imaging and Sensing 2009, 71772I (24 February 2009); doi: $10.1117 / 12.809327$

SPIE. Event: SPIE BiOS, 2009, San Jose, California, United States 


\title{
Monitoring the Healing Process of Laser-Induced Microvascular Lesions Using Optical-Resolution Photoacoustic Microscopy
}

\author{
Song Hu, Konstantin I. Maslov, and Lihong V. Wang \\ Optical Imaging Laboratory, Department of Biomedical Engineering, Washington University in St. \\ Louis, St. Louis, Missouri, USA 63130
}

\begin{abstract}
Optical resolution photoacoustic microscopy (OR-PAM) possesses optical resolution and reveals endogenous optical absorption contrast, promising to be a valuable tool for in vivo microvascular imaging. In laser dermatology, OR-PAM can provide fruitful structural and functional information about the targeted microvascular lesions, such as their threedimensional (3D) morphology, precise location inside the tissue, and blood oxygenation within single vessels, which will facilitate accurate diagnosis and proper treatment. More importantly, the advantages of noninvasiveness and measurement consistency also permit OR-PAM to monitor the healing process of the laser-surgical wound noninvasively. In this work, we employed OR-PAM to monitor the healing process of microvascular lesions induced by nanosecond-pulsed laser. Our results indicate that OR-PAM could be a very useful tool in laser dermatology and laser microsurgery.
\end{abstract}

Keywords: Optical-resolution photoacoustic microscopy, vascular lesion, wound healing, longitudinal monitoring.

\section{INTRODUCTION}

Common cutaneous vascular lesions include port wine stains, telangiectasia, capillary hemangioma and other vascular malformations. Since the laser wavelength and pulse width need to be optimized to selectively damage the targeted vascular lesions with minimum effect to the surrounding tissues ${ }^{1}$, prompt and accurate diagnosis of these lesions is essential for proper treatment. Moreover, the biological repair of such highly specific damage needs to be understood ${ }^{1}$ to fully evaluate the treatment.

However, until now, there has not been a technique that could provide chronic imaging of the intact microcirculation. Interferences and side-effects due to invasive procedures and exogenous contrast agents affect the measurement accuracy and impede longitudinal studies.

The advantages of photoacoustic microscopy (PAM) over traditional optical and ultrasonic imaging include primarily the detection of endogenous optical absorption contrast at ultrasonic resolutions ${ }^{2,3}$. In PAM, a pulsed laser beam is weakly focused into the biological tissue to produce emission of ultrasonic waves due to thermal expansion of the medium caused by absorbed laser radiation. The ultrasonic waves are then detected with a focused ultrasonic transducer to form high-resolution volumetric images. Among the existing photoacoustic imaging technologies, the spatial resolutions depend almost solely on the ultrasonic parameters including the frequency and the numerical aperture (NA). For example, in the recently developed dark-field confocal PAM, a lateral resolution of $50 \mu \mathrm{m}$ was achieved with a center frequency of $50 \mathrm{MHz}$ and an NA of $0.44^{2-4}$. This resolution is adequate for many biomedical applications. However, to resolve the distal functional units in the microcirculation, such as capillaries with $4-9 \mu \mathrm{m}$ diameters, higher spatial resolution is required.

If both the lateral and axial resolutions are provided ultrasonically, the imaging penetration is limited by the frequencydependent ultrasonic attenuation in tissue. So we have developed optical-resolution photoacoustic microscopy (ORPAM) to achieve micrometer lateral resolution by using diffraction-limited optical focusing ${ }^{5,6}$. Although having a

Photons Plus Ultrasound: Imaging and Sensing 2009, edited by Alexander A. Oraevsky, Lihong V. Wang, Proc. of SPIE Vol. 7177, 71772I - @ 2009 SPIE · CCC code: 1605-7422/09/\$18 - doi: 10.1117/12.809327 
comparable depth penetration limit with existing high-resolution optical imaging modalities (including optical confocal microscopy, two-photon microscopy, and optical coherence tomography), OR-PAM is primarily sensitive to optical absorption contrast, whereas the other modalities are dominantly sensitive to optical scattering or fluorescence contrast.

\section{METHODS AND MATERIALS}

\subsection{OR-PAM system}

Our OR-PAM system (Fig. 1) employs optical focusing to achieve micrometer-level lateral resolution. A dye laser pumped by an Nd:YLF laser is used as the excitation source. Laser pulses (pulse duration: $7 \mathrm{~ns}$ ) from the dye laser are spatially filtered by a $25-\mu \mathrm{m}$-diameter pinhole. The pinhole is then imaged to a diffraction-limited optical focal spot with a diameter of $3.7 \mu \mathrm{m}$ by an objective lens (NA: 0.1 ). The laser pulse energy after the objective lens is measured to be 80 nJ. Ultrasonic focusing is achieved through a plano-concave lens (radius of curvature: $5.2 \mathrm{~mm}$; aperture: $6.4 \mathrm{~mm}$; NA in water: 0.46$)$ and the ultrasonic focal diameter is $27 \mu \mathrm{m}$. The optical objective lens and the ultrasonic transducer are configured coaxially and confocally as shown in Fig. 1.

The combination of time-resolved detection of the photoacoustic waves with a two-dimensional (2D) raster scanning along the $x-y$ plane generates volumetric dataset, which can be viewed through direct volumetric rendering, crosssectional (B-scan) images, or maximum amplitude projection (MAP) images.

The lateral resolution and the imaging depth of the OR-PAM system were experimentally estimated to be better than 5 $\mu \mathrm{m}$ and more than $700 \mu \mathrm{m}$, respectively ${ }^{6}$. The axial resolution was calculated to be $15 \mu \mathrm{m}$ based on the manufactoryspecified bandwidth of the ultrasonic transducer.

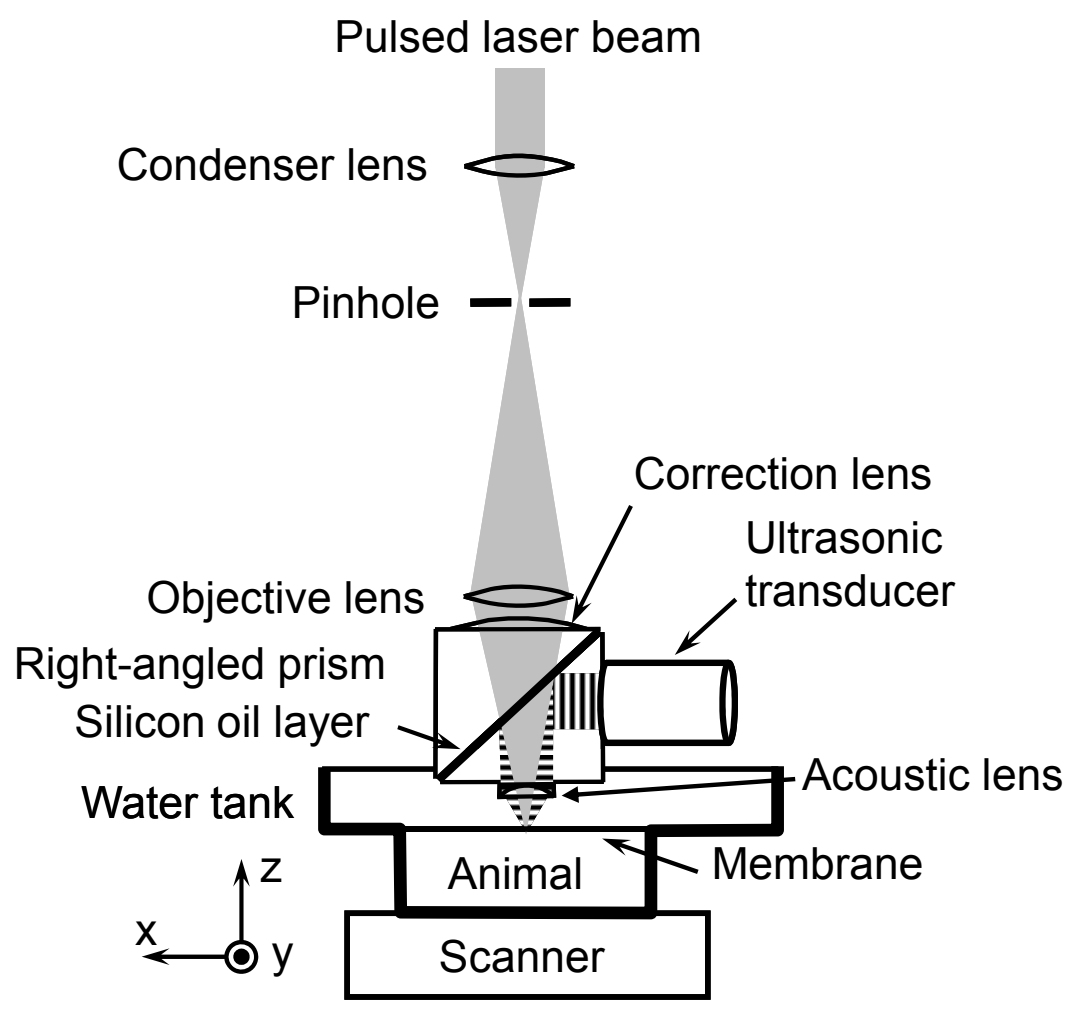

Figure 1. Schematic of the OR-PAM system. 


\subsection{Animal preparation}

All experimental animal procedures were carried out in conformance with the laboratory animal protocol approved by the School of Medicine Animal Studies Committee of Washington University in St. Louis.

Nude mice (Hsd:Athymic Nude-Foxn 1NU, Harlan Co.) were used. Before imaging, the hair on the ears was gently removed with human hair-removing lotion. A dose of $87 \mathrm{mg} / \mathrm{kg}$ Ketamine and $13 \mathrm{mg} / \mathrm{kg}$ Xylazine was administered intraperitoneally to anesthetize the animal, and anesthesia was maintained throughout the experiments using an isoflurane machine (1.0-1.5\% vaporized isoflurane with an airflow rate of 1 liter $/ \mathrm{min})$. The body temperature of the animal was maintained at $37^{\circ} \mathrm{C}$ with a temperature controlled heating pad. At the end of the experiment, the animals were killed by an intraperitoneal administration of pentobarbital at a dosage of $100 \mathrm{mg} / \mathrm{kg}$.

\subsection{Laser induced microvascular lesion}

For wound healing experiment, we randomly selected a 1-mm-by-1-mm region in a nude mouse ear via a commercial transmission-mode optical microscope, and then imaged the selected region using our OR-PAM system. After that, we maximized the output of the nanosecond pulsed laser and removed the pinhole for destruction. The central part of the selected region was scanned with the high-intensity laser beam for continuously ten minutes. The selected region was monitored right after the laser irradiation and in the subsequent four days to record the wound healing process.

\section{RESULTS AND DISCUSSION}

Prior to performing the laser destruction, the microvasculature of the nude mouse ear was imaged in vivo noninvasively (Fig. 2). The OR-PAM image of the microvascular network in Fig. 2(B) is highly correlated with the photograph in Fig. 2(A) taken by the transmission-mode optical microscope at a $4 \times$ magnification. However, small vessels as well as capillaries are only observable to OR-PAM. The mean ratio of the photoacoustic amplitudes between the blood vessels and the background is $20: 1$, which demonstrates a high endogenous optical absorption contrast ${ }^{6}$. Some microvessels with diameters of $\sim 5 \mu \mathrm{m}$ in Fig. 2(B) are expected to be single capillaries.

Throughout the whole wound healing monitoring process, $570 \mathrm{~nm}$ laser wavelength - a specific optical wavelength at which the molar extinction coefficients of the oxy- and deoxy-hemoglobin are equal-is selected, the photoacoustic signal represents the total hemoglobin concentration, regardless of the hemoglobin oxygenation.

The duration of the laser pulses used to destruct the microvessels is $\sim 7 \mathrm{~ns}$, which is much smaller than the thermal relaxation time. These short pulses generate high local peak temperature, potentially leading to intravascular cavitation from explosive vaporization that may result in vessel rupture and sequential hemorrhage ${ }^{7}$. As shown in Fig. 3, right after the laser destruction, the irradiated blood vessels were ruptured and red blood cells were popped out to form hemorrhages.

After twenty-four hours, a large portion of the hemorrhage was washed out and the residual hemorrhages were cleared gradually during the sequential days.

Inflammation induced vasodilation was observed one day after the injury and lasted throughout the five-day monitoring. Inflammation represents the actions of cleansing the wound of infection, releasing soluble mediators, and generating growth factors that are involved in the recruitment and activation of fibroblasts and epithelial cell to restore the damaged microcirculation ${ }^{8}$.

After four days, the pulsed laser induced microvascular lesion recovered from blood coagulation and hemostasis. However, the vessel diameters were still larger than those in the normal status, which implied the presence of inflammation. This observation is in good agreement with the known physiological knowledge, which suggests a inflammatory phase of up to two weeks 8 . 
A

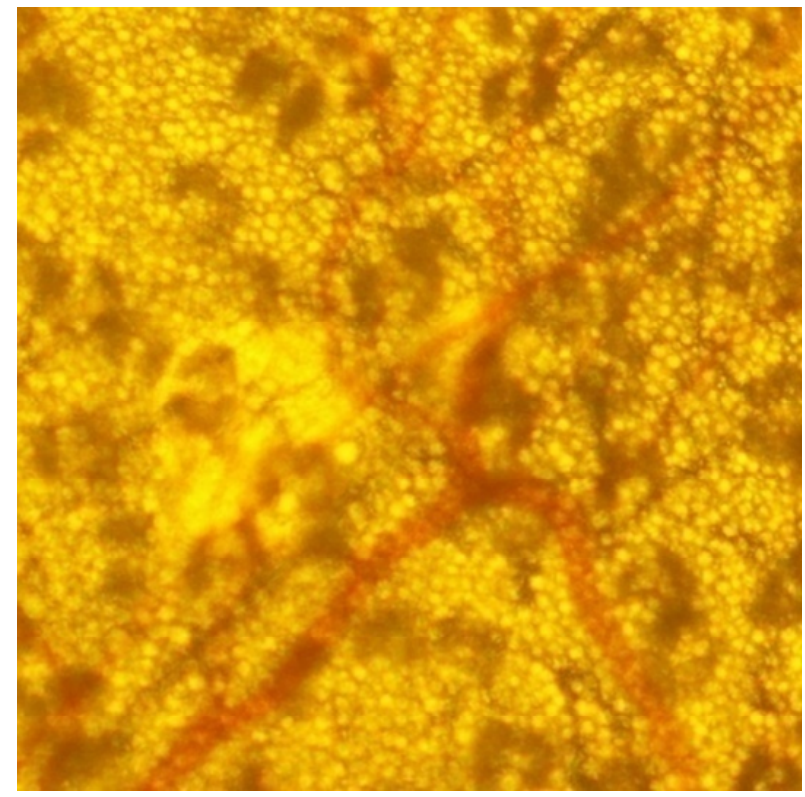

B

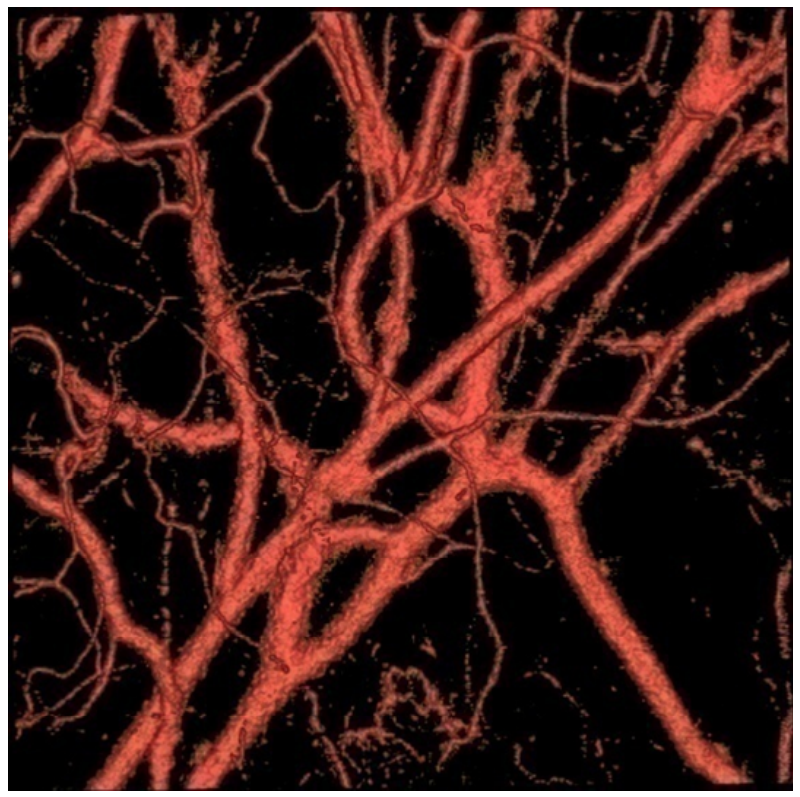

\section{$-50 \mu \mathrm{m}$}

Figure 2. The healing process of a pulsed laser induced microvascular lesion-before the laser destruction. (A) The photograph taken by a commercial transmission-mode optical microscope; (B) The front-view of the 3D microvascular morphology acquired by OR-PAM at $570 \mathrm{~nm}$.

A

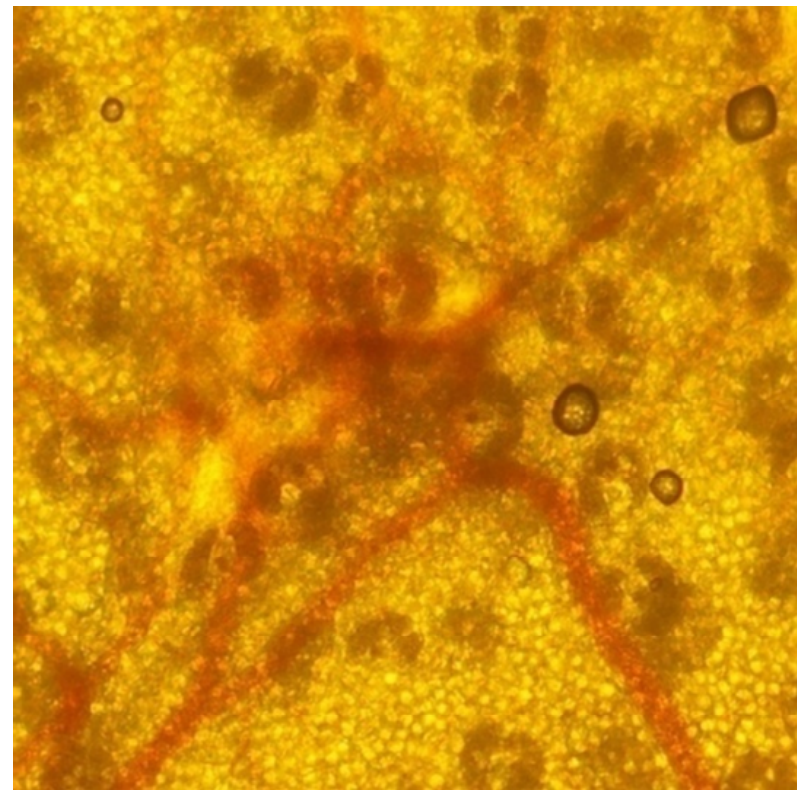

B

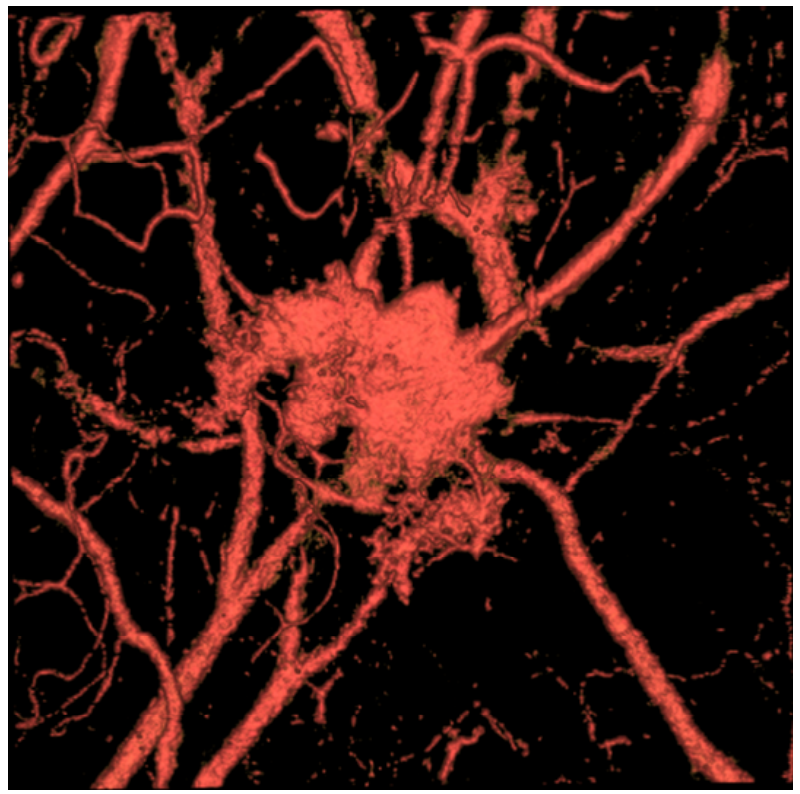

$-50 \mu \mathrm{m}$

Figure 3. The healing process of a pulsed laser induced microvascular lesion-right after the laser destruction (Day 0). (A) The photograph taken by a commercial transmission-mode optical microscope; (B) The front-view of the 3D microvascular morphology acquired by OR-PAM at $570 \mathrm{~nm}$. 
A

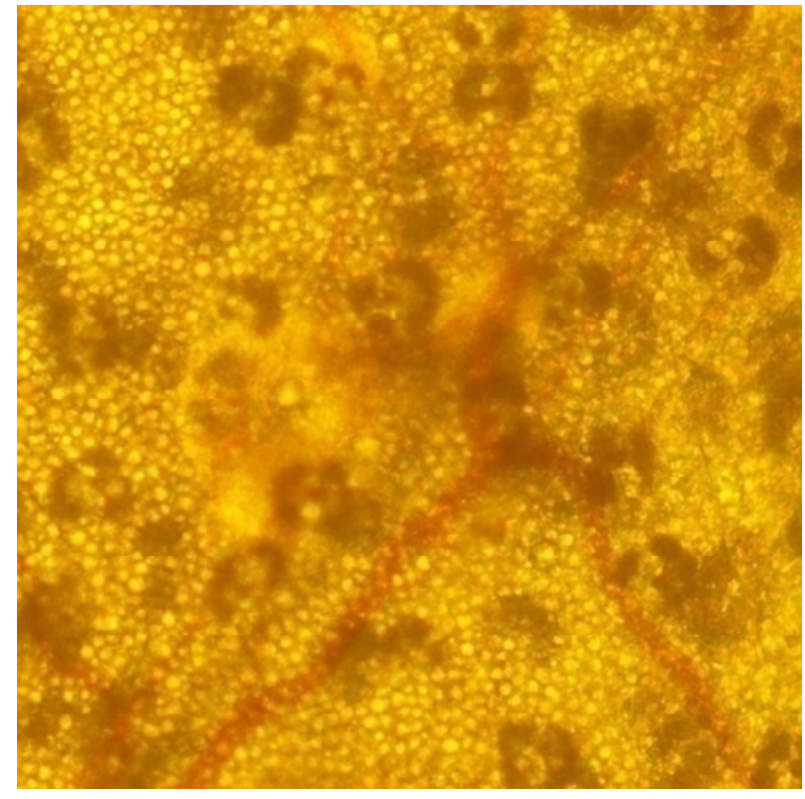

B

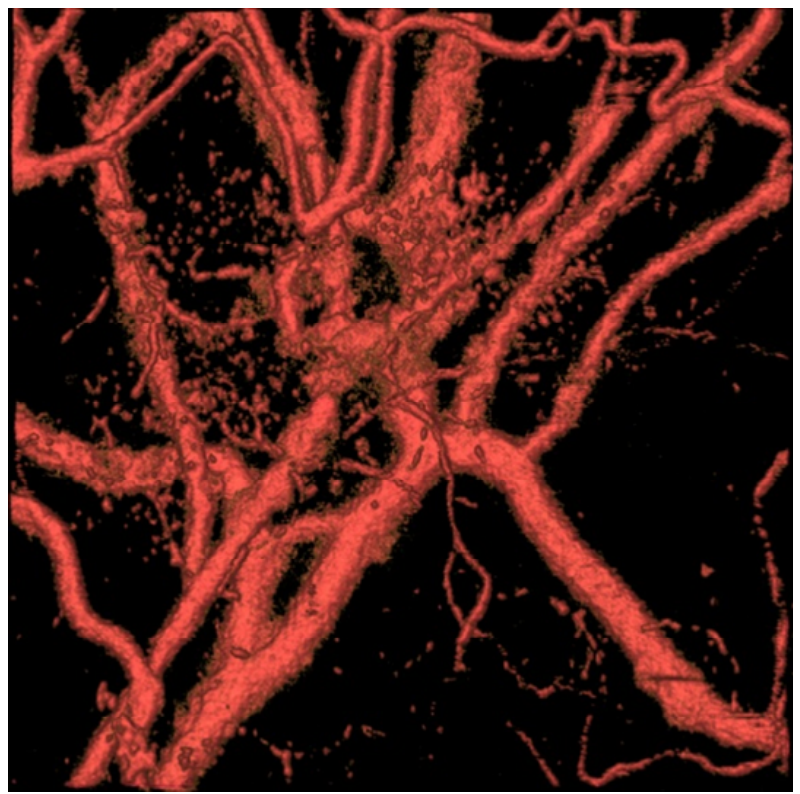

\section{$-50 \mu \mathrm{m}$}

Figure 4. The healing process of a pulsed laser induced microvascular lesion-Day 1. (A) The photograph taken by a commercial transmission-mode optical microscope; (B) The front-view of the 3D microvascular morphology acquired by OR-PAM at $570 \mathrm{~nm}$.

A

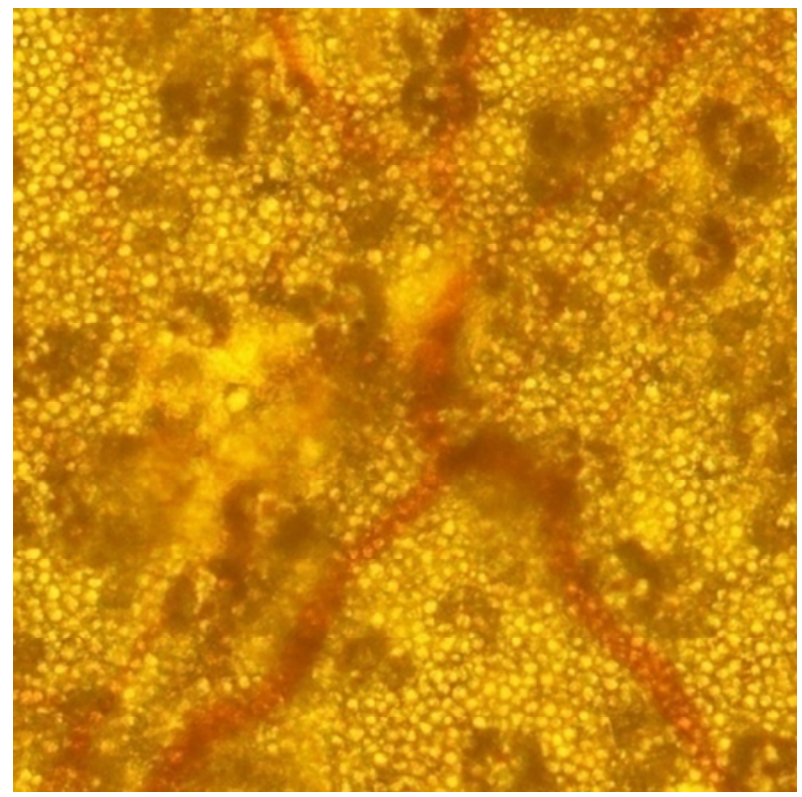

B

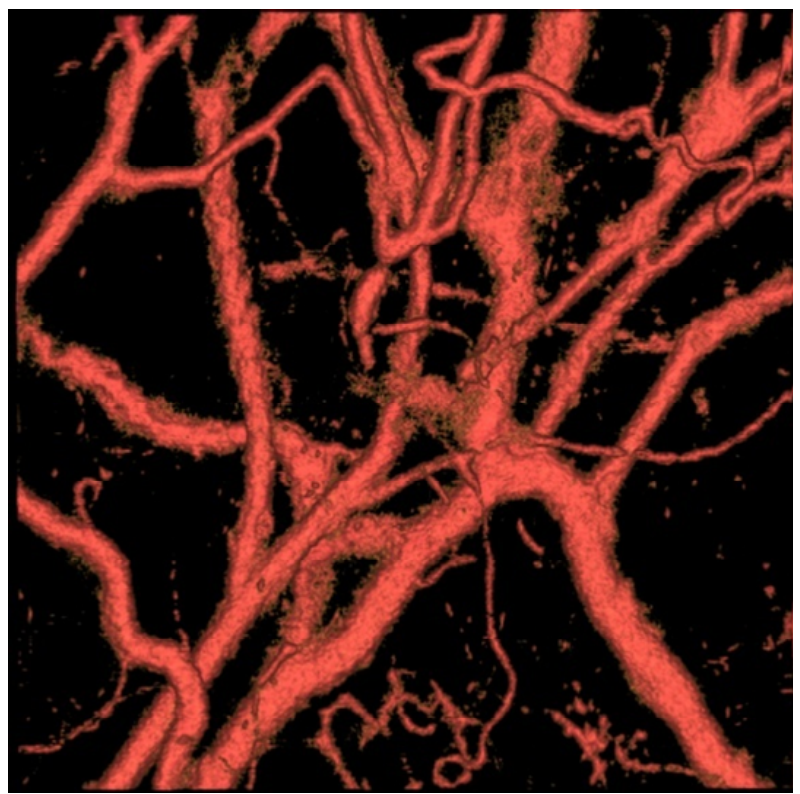

$-50 \mu \mathrm{m}$

Figure 5. The healing process of a pulsed laser induced microvascular lesion-Day 2. (A) The photograph taken by a commercial transmission-mode optical microscope; (B) The front-view of the 3D microvascular morphology acquired by OR-PAM at $570 \mathrm{~nm}$. 
A

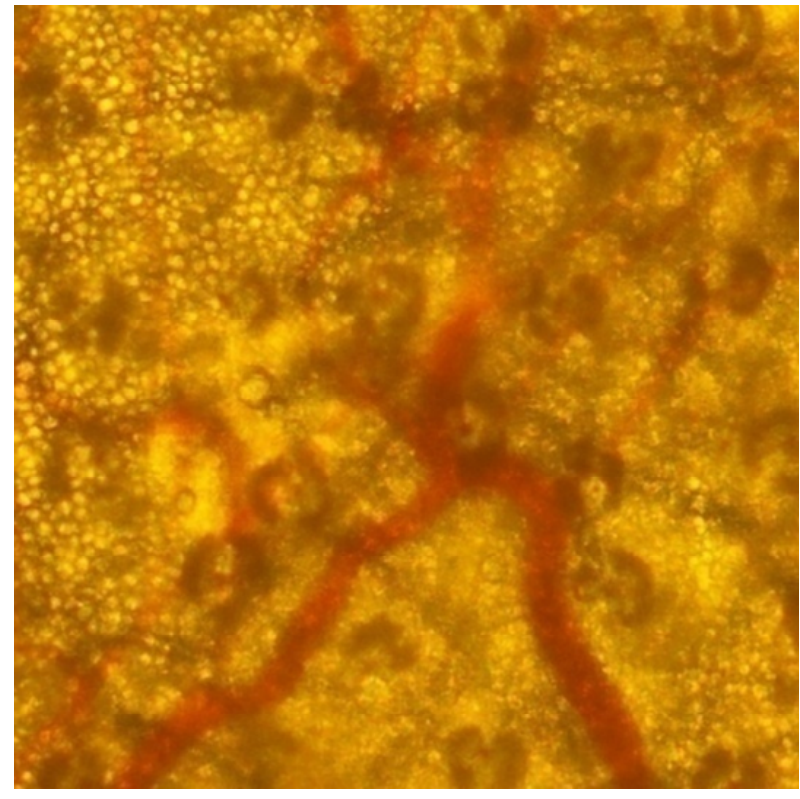

B

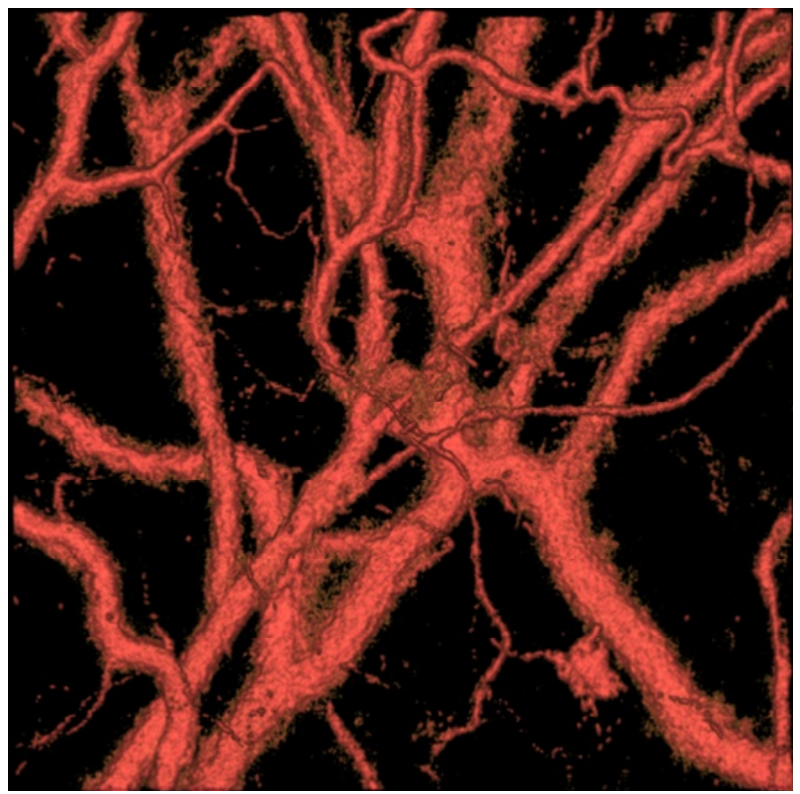

\section{$-50 \mu \mathrm{m}$}

Figure 6. The healing process of a pulsed laser induced microvascular lesion-Day 3. (A) The photograph taken by a commercial transmission-mode optical microscope; (B) The front-view of the 3D microvascular morphology acquired by OR-PAM at $570 \mathrm{~nm}$.

A

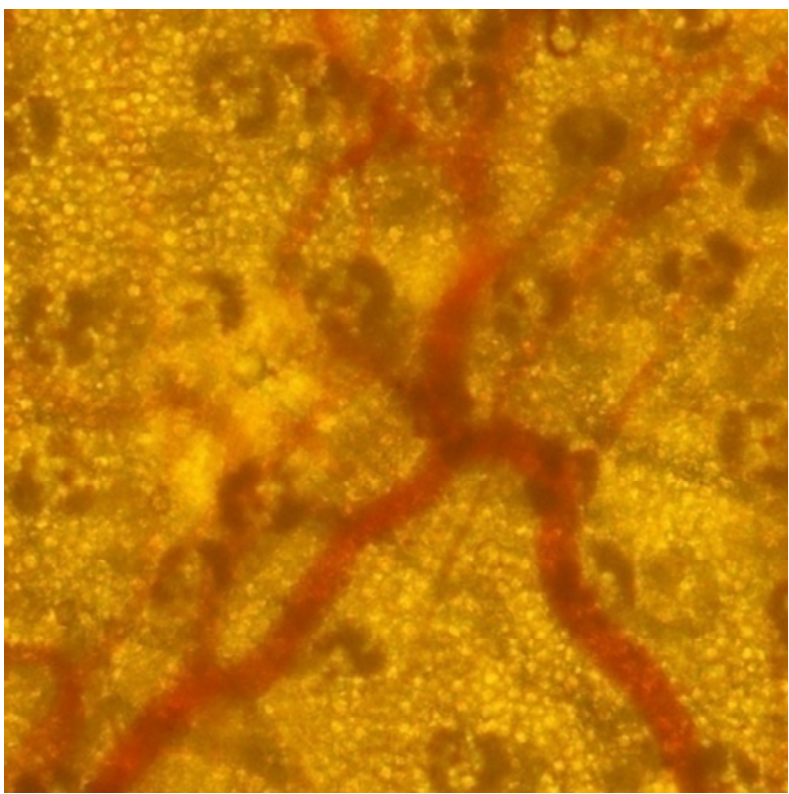

B

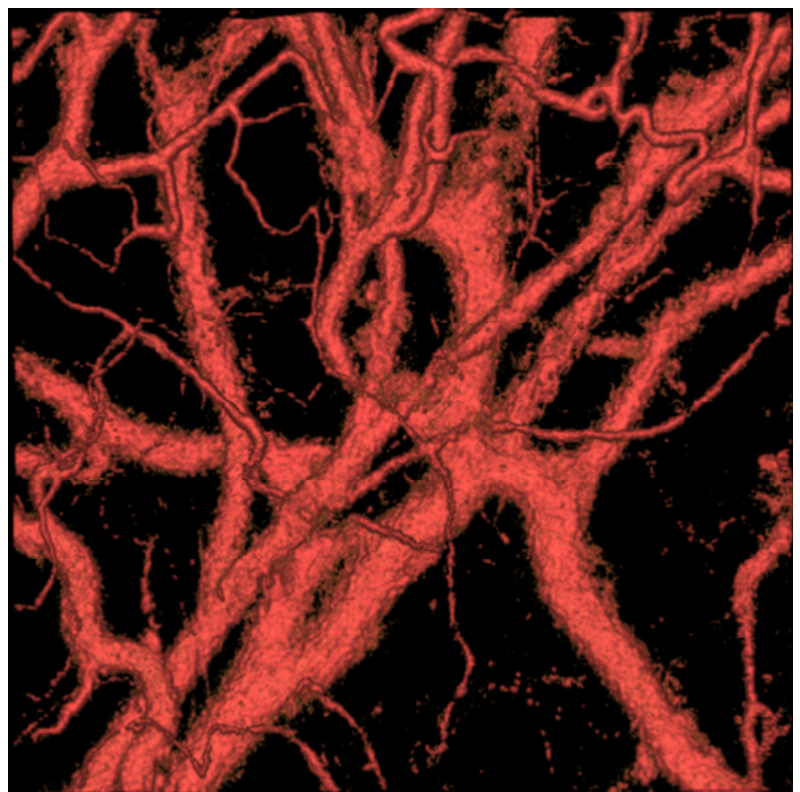

$-50 \mu \mathrm{m}$

Figure 7. The healing process of a pulsed laser induced microvascular lesion-Day 4. (A) The photograph taken by a commercial transmission-mode optical microscope; (B) The front-view of the 3D microvascular morphology acquired by OR-PAM at $570 \mathrm{~nm}$. 


\section{CONCLUSIONS}

The healing process of microvascular lesions induced by a nanosecond pulsed laser was monitored longitudinally by both our OR-PAM system and a commercial transmission-mode optical microscope. Our experimental observation is in good agreement with that of the commercial transmission-mode optical microscope but provides more detailed morphological information. Moreover, our observation is confirmed by the known physiological knowledge. OR-PAM has potentially important applications in laser dermatology and laser microsurgery.

\section{ACKNOWLEDGEMENTS}

This work was sponsored by National Institutes of Health grants R01 EB000712, R01 NS46214 (Bioengineering Research Partnerships), R01 EB008085, and U54 CA136398 (Network for Translational Research). L.W. has a financial interest in Endra, Inc., which, however, did not support this work.

\section{REFERENCES}

1. Anderson, R.R. \& Parrish, J.A. Selective photothermolysis: precise microsurgery by selective absorption of pulsed radiation. Science 220, 524-527 (1983).

2. Maslov, K., Stoica, G. \& Wang, L.V. In vivo dark-field reflection-mode photoacoustic microscopy. Optics letters 30, 625-627 (2005).

3. Zhang, H.F., Maslov, K., Stoica, G. \& Wang, L.V. Functional photoacoustic microscopy for high-resolution and noninvasive in vivo imaging. Nature biotechnology 24, 848-851 (2006).

4. Zhang, H.F., Maslov, K. \& Wang, L.V. In vivo imaging of subcutaneous structures using functional photoacoustic microscopy. Nature protocols 2, 797-804 (2007).

5. Maslov, K., Zhang, H.F., Hu, S., \& Wang, L.V. Optical-resolution confocal photoacoustic microscopy. Proc. SPIE 6856, 68561I (2008).

6. Maslov, K., Zhang, H.F., Hu, S. \& Wang, L.V. Optical-resolution photoacoustic microscopy for in vivo imaging of single capillaries. Optics letters 33, 929-931 (2008).

7. Suthamjariya, K., Farinelli, W.A., Koh, W. \& Anderson, R.R. Mechanisms of microvascular response to laser pulses. The Journal of investigative dermatology 122, 518-525 (2004).

8. Chin, G.A., Diegelmann, R.F. \& Schultz, G.S. Cellular and molecular regulation of wound healing Edn. 1st. (Talyor \& Francis, Boca Raton; 2005). 